\title{
Diabetes mellitus y atrofia óptica: estudio del síndrome de Wolfram
}

\author{
Bernardette Rivas-Gómez y Alfredo Adolfo Reza-Albarrán \\ Departamentos de Medicina Interna y Endocrinología, Instituto Nacional de Ciencias Médicas y Nutrición Salvador Zubirán, Ciudad de México, \\ México
}

\section{Resumen}

Antecedentes: El síndrome de Wolfram (SW), también conocido por el acrónimo DIDMOAD, es una enfermedad hereditaria rara y progresiva, de transmisión autosómica recesiva, cuyos criterios diagnósticos mínimos son diabetes mellitus y atrofia óptica antes de los 15 años de edad. Objetivo: Describir la presentación clínica, bioquímica y molecular del SW en un hospital de tercer nivel en México. Métodos: Se revisaron los expedientes de pacientes que cumplían con criterios diagnósticos clínicos mínimos de SW atendidos entre enero de 1987 y mayo de 2015 en un hospital de tercer nivel en México. Resultados: Cinco pacientes cumplieron con los criterios de inclusión (tres hombres y dos mujeres). La diabetes mellitus fue la primera manifestación del síndrome en todos ellos, con una media de edad al diagnóstico de $5.8 \pm 2.71$ años, mientras que el diagnóstico del SW se estableció en promedio a los $15.8 \pm 8.37$ años. Todos los pacientes tenían atrofia óptica y dos presentaron el espectro DIDMOAD completo. Se describen nuevas asociaciones con hepatitis autoinmunitaria y cáncer de testículo. Conclusiones: El presente estudio muestra la variabilidad de presentación clínica del SW y dos asociaciones no descritas previamente.

PALABRAS CLAVE: Síndrome de Wolfram. DIDMOAD. Retículo endoplásmico. Diabetes mellitus. Atrofia óptica.

\begin{abstract}
Background: Wolfram syndrome (WS), also known by the acronym DIDMOAD, is a rare and progresive hereditary disease of autosomal recessive inheritance which minimum ascertainment diagnostic criteria are the occurrence together of diabetes mellitus and optic atrophy before 15 years of age. Objective: To describe the clinical, biochemical and molecular profile of WS in a tertiary care hospital in Mexico. Methods: We reviewed patients records who fulfill the minimum ascertainment diagnostic criteria of WS presenting between January 1987 and May 2015 in a tertiary care hospital in Mexico. Results: Five patients fulfill the inclusion criteria (three male and two female). Diabetes mellitus was the first manifestation of the syndrome in all of them, with a mean age at diagnosis of $5.8 \pm 2.71$ years, while the WS diagnosis was established at a mean age of $15.8 \pm$ 8.37 years. All the patients had optic atrophy and two of them presented with the complete DIDMOAD spectrum. We found new associations with autoimmune hepatitis and testicular cancer. Conclusions: This study shows the variability of clinical presentation of WS, as well as two new associations.
\end{abstract}

KEY WORDS: Wolfram syndrome. DIDMOAD. Endoplasmic reticulum. Diabetes mellitus. Optic atrophy. 


\section{Introducción}

El síndrome de Wolfram (SW) (MIM 222300), descrito por primera vez en 1938 por Wolfram y Wagener $^{1}$, también se conoce como DIDMOAD por sus siglas en inglés (Diabetes Insipidus, Diabetes Mellitus, Optic Atrophy, and Deafness), aunque muchos proponen que debería ser llamado DIDMOADUD, haciendo referencia estas dos últimas siglas a la alta frecuencia con que se presentan alteraciones urológicas (Urinary Disfunction)2,3.

Inicialmente se creía que deleciones o mutaciones en el genoma mitocondrial (mtADN) eran las causantes de este síndrome debido a las similitudes fenotípicas de pacientes con SW y enfermedades mitocondriales ${ }^{4-6}$; sin embargo, la presencia de hijos afectados y no afectados de padres sanos, frecuentemente consanguíneos, sugirieron el modo de herencia autosómico recesivo con penetrancia incompleta y expresividad variable ${ }^{7}$. No fue hasta 1998 que se identificó el gen WFS1, causante del SW, en el cromosoma $4 \mathrm{p} 16.1^{8}$. Este gen está formado por ocho exones y codifica una proteína tetramérica llamada wolframina, que está formada por 890 aminoácidos y que se localiza en la membrana del retículo endoplásmico $(R E)$, expresándose en valores muy altos en el cerebro, el páncreas, el corazón y el músculo esquelético ${ }^{9}$.

La tasa de síntesis, plegamiento y transporte de proteínas está coordinada por un eficiente sistema de calidad de control, que asegura que solo las proteínas adecuadamente dobladas puedan salir del RE para ejercer su función biológica ${ }^{10}$. Las proteínas que no son dobladas adecuadamente se retienen en el RE o son sometidas a degradación. La homeostasis del RE es definida como el estado de equilibrio entre la demanda celular para la síntesis de proteínas y la capacidad de plegamiento del RE para promover el transporte y la maduración proteica para satisfacer dicha demanda ${ }^{11}$. Las proteínas no plegadas o mal plegadas tienen un efecto deletéreo en la función celular y pueden causar su muerte, proceso al que se conoce como proteotoxicidad o estrés del $\mathrm{RE}^{10,12-15}$.

Para mantener la homeostasis del RE, las células han desarrollado un mecanismo adaptativo de respuesta al estrés, llamado respuesta a proteínas desplegadas, o UPR por sus siglas en inglés (Unfolded Protein Response), que comprende un conjunto de vías de señalización intracelular para hacer frente al estrés metabólico, oxidativo e inflamatorio ${ }^{10,16,17}$. La
UPR tiene la capacidad de actuar como un switch binario entre la vida y la muerte, pues puede regular tanto los efectores de supervivencia como los apoptóticos ${ }^{18}$. Existen dos tipos de estrés sobre la célula: el tolerable (o que es capaz de solucionar) y el que no. Cuando el estrés es solucionable, se ponen en marcha todas las cascadas y mecanismos para promover la supervivencia, mientras que cuando la UPR detecta un estrés que no es capaz de resolver, o cuando sus mecanismos son incapaces de restaurar la homeostasis del RE ante estresores crónicos, hay una hiperactivación de la UPR que desencadena la acción de efectores de muerte celular que llevan a la apoptosis ${ }^{11}$.

La wolframina es un componente importante de la vía de supervivencia de la UPR ${ }^{10,18}$. En el SW, la deficiencia de wolframina causa hiperactivación y un alza sin control de vías de señalización celular que causan la muerte por apoptosis en las células sensibles ${ }^{11,19}$.

Estudios clínicos y genéticos han demostrado que la disfunción heredada o adquirida del RE es causante o contribuyente para la aparición de enfermedades mucho más comunes que el SW, como la diabetes mellitus tipo 1 y tipo 2, la obesidad, la aterosclerosis, la dislipidemia, la esteatosis hepática, la enfermedad inflamatoria intestinal, muchos tipos distintos de cáncer y enfermedades neurodegenerativas como la enfermedad de Alzheimer, la corea de Huntington y enfermedades por priones, por lo que el RE se convierte en un blanco terapéutico atractivo para estas enfermedades ${ }^{10,20-23}$; sin embargo, el SW es considerado como el prototipo de las enfermedades del RE.

La prevalencia del SW se estima en 1 por 770,000 personas en el Reino Unido, con una prevalencia de portador de 1 en $354^{24}$, de 1 en 710,000 en la población japonesa ${ }^{25}$ y de 1 en 805,000 en la India ${ }^{26}$. Se han descrito prevalencias más altas, como 1 en 68,000 personas en Líbano ${ }^{27}$, parcialmente atribuible a las altas tasas de consanguinidad en esta región.

El diagnóstico de SW continúa siendo clínico, utilizando el análisis mutacional para confirmar el diagnóstico ${ }^{4}$. Los criterios mínimos para el diagnóstico son diabetes mellitus y atrofia óptica, ambos con inicio antes de los 15 años de edad, con un valor predictivo positivo del $83 \%$ y negativo del $1 \%{ }^{24}$.

La presentación tardía, con inicio de diabetes y atrofia óptica después de los 15 años de edad, tiene mucha variabilidad en su frecuencia en las distintas cohortes; sin embargo, estas diferencias pueden deberse a un infradiagnóstico de pacientes con SW mayores de 15 años y a una diferencia en las distintas series en el estudio de fenotipos atípicos ${ }^{6,28-30}$. 
La edad promedio de muerte de los pacientes con SW es a los 30 años, y la principal causa de muerte es la falla respiratoria a causa de la atrofia del tallo cerebral $^{31}$. Otras causas importantes de morbimortalidad son las infecciones del tracto urinario y la insuficiencia renal crónica.

Existe muy poca información publicada sobre el SW en México. Conocer estos datos ayudará a optimizar la infraestructura para el estudio y manejo de los pacientes con SW, pudiendo influir de manera favorable en su diagnóstico, evolución, calidad de vida, rehabilitación y pronóstico.

En este estudio describimos las características clínicas, bioquímicas y moleculares (en quienes esta última se encuentre disponible) del SW en un hospital de tercer nivel.

\section{Métodos}

Este estudio es retrospectivo, transversal y descriptivo. Se solicitó al archivo clínico la relación de pacientes con diagnósticos de diabetes asociada a atrofia óptica (de acuerdo con los códigos de la CIE 10) en el periodo comprendido entre el 1 de enero de 1987 y el 31 de mayo de 2015. Se revisaron los expedientes y se excluyeron aquellos en quienes ya se había descartado el SW y aquellos en los que la atrofia óptica se atribuyó a alguna otra causa distinta al SW. La información se capturó en una base de datos y el análisis de variables se realizó mediante STATA versión 13.

\section{Resultados}

Tras la búsqueda de expedientes que contaban con los diagnósticos de diabetes y atrofia óptica se obtuvieron 11 registros, de los cuales se excluyeron seis de acuerdo con los criterios de exclusión y eliminación. Incluimos a los cinco pacientes restantes y revisamos los expedientes para la recolección y el análisis de datos. De estos, el $40 \%(n=2)$ eran mujeres y el $60 \%(n=3)$ eran hombres. La edad promedio al diagnóstico de la diabetes fue de $5.8 \pm 2.71$ años y en todos los casos se trató de la primera manifestación del síndrome.

La edad promedio al diagnóstico de la atrofia óptica fue de $12.2 \pm 6.37$ años, mientras que el diagnóstico de SW se estableció en promedio a los $15.8 \pm 8.37$ años de edad.

Ninguno de los pacientes incluidos en este estudio tenía antecedentes de consanguinidad ni heredofamiliares que sugirieran SW en generaciones anteriores.

Ningún paciente se encontraba en metas de control de la diabetes; el promedio de la última hemoglobina glucosilada fue de $8.44 \%(7.2-10.8 \%)$ y todos se encontraban bajo esquema intensivo de insulina con tres o más inyecciones al día y con dosis promedio de 0.64 unidades $/ \mathrm{kg}$, con un rango de 0.29 a 1.08 unidades $/ \mathrm{kg}$.

El índice de masa corporal promedio de los sujetos del estudio fue de $21.74 \pm 2.92 \mathrm{~kg} / \mathrm{m}^{2}$. En la Tabla 1

Tabla 1. Variables estudiadas divididas por sexo

\begin{tabular}{lccc}
\hline Variable & & Media (DE) & Mujeres \\
\cline { 2 - 4 } & Hombres y mujeres & Hombres & $51.35(9.40)$ \\
\hline Peso (kg) & $55.34(11.27)$ & $58(15.71)$ & $1.50(0.098)$ \\
Talla (metros) & $1.58(0.092)$ & $1.64(0.069)$ & $22.55(1.06)$ \\
IMC (kg/m²) & $21.74(2.61)$ & $21.2(3.93)$ & $4(4.24)$ \\
$\begin{array}{l}\text { Edad al diagnóstico } \\
\text { de DM (años) }\end{array}$ & $5.8(2.71)$ & $7(2)$ & $14(9.89)$ \\
$\begin{array}{l}\text { Edad al diagnóstico } \\
\text { de AO (años) }\end{array}$ & $12.2(6.37)$ & $11(5.19)$ & $24.5(0.70)$ \\
$\begin{array}{l}\text { Edad al diagnóstico } \\
\text { de SW (años) }\end{array}$ & $15.8(8.37)$ & $10(7)$ & $22.5(6.36)$ \\
$\begin{array}{l}\text { Tiempo de evolución } \\
\text { de DM (años) }\end{array}$ & $18.8(4.26)$ & $16.3(1.52)$ & \\
$\begin{array}{l}\text { Hemoglobina } \\
\text { glucosilada (\%) }\end{array}$ & $8.44(1.31)$ & $7.53(0.49)$ & $9.8(1.41)$ \\
\hline AO: atrofia óptica; DE: desviación estándar; DM: diabetes mellitus; IMC: índice de masa corporal; SW: síndrome de Wolfram. &
\end{tabular}


se muestran algunas de las variables estudiadas divididas por sexo.

De los cinco pacientes, dos tenían diagnóstico de diabetes insípida central, tres tenían alteraciones auditivas de moderadas a graves, cuatro tenían al menos alguna alteración neurodegenerativa, cuatro presentaban manifestaciones psiquiátricas, cinco tenían alteraciones urológicas, cinco tenían alteraciones endocrinológicas y tres tenían alteraciones gastrointestinales. Dos de los pacientes tenían el espectro DIDMOAD completo y los cinco continuaban vivos al momento del estudio, por lo que no fue posible estudiar la mortalidad.

Solo un paciente tenía solicitado un estudio de neuroimagen, una tomografía computarizada simple de cráneo, que no mostró alteraciones y que había sido solicitada por motivos distintos al SW.

\section{Discusión}

El SW es una enfermedad rara con gran pleomorfismo fenotípico. La diabetes en el SW es deficiente en insulina, similar a la diabetes mellitus tipo 1, pero no autoinmunitaria y no ligada al complejo mayor de histocompatibilidad. Su causa es una degeneración selectiva de las células beta que se inicia prácticamente desde el nacimiento, por lo que suele presentarse a edades más tempranas que la diabetes de causa autoinmunitaria, con una edad promedio al diagnóstico de 6 años ${ }^{32,33}$, de modo similar a lo observado en nuestra población, en la que la edad al diagnóstico fue de $5.8 \pm 2.71$ años. Como en todos nuestros pacientes, suele ser la primera manifestación del síndrome hasta en el $80 \%$ de los casos. Ninguno de los pacientes de este estudio tenía determinación de anticuerpos para excluir un origen autoinmunitario.

Diversos estudios han demostrado una progresión más lenta de la diabetes, con una menor incidencia de complicaciones microvasculares, cetoacidosis diabética y variabilidad glucémica, así como menos requerimientos de insulina en comparación con pacientes con diabetes tipo $1^{17,34,35}$. En nuestro estudio, solo una paciente tenía antecedente de un cuadro de cetoacidosis diabética y solo dos pacientes tenían documentada alguna complicación microvascular (retinopatía diabética no proliferativa), a pesar de tener un tiempo promedio de evolución de la diabetes de $18.8 \pm 4.26$ años. Ningún paciente tuvo diagnóstico de nefropatía diabética según la determinación de albuminuria del último año de seguimiento.
Si bien se han descrito casos de diagnóstico de atrofia óptica en rangos tan amplios como de 6 semanas a 30 años de edad ${ }^{3,36}$, en promedio esta se diagnostica a los 11 años de edad, provocando amaurosis aproximadamente 8 años después del diagnóstico. En nuestro estudio, la edad promedio al diagnóstico fue de $12.2 \pm 6.37$ años y tres de los pacientes ya tenían amaurosis; de manera semejante a la mayoría de los pacientes de otras series, en todos fue la segunda manifestación del síndrome. En cuanto a otras alteraciones of talmológicas, únicamente se encontró catarata bilateral en una de las pacientes.

La diabetes insípida central, secundaria al proceso neurodegenerativo del hipotálamo y la hipófisis posterior, se presenta en el $51-87 \%$ de los pacientes con SW, con una edad promedio al diagnóstico de 14 años, siendo la poliuria su primera manifestación clínica ${ }^{37-39}$. En el presente estudio, dos pacientes (40\%) presentaron diabetes insípida central, con una edad promedio al diagnóstico de 17 años; ambos se encontraban en tratamiento con desmopresina.

Dependiendo de la serie, las alteraciones auditivas se presentan en el $62-100 \%$ de los pacientes con $\mathrm{SW}^{30,40}$. La pérdida auditiva suele ser lentamente progresiva y afecta sobre todo las frecuencias altas, entre 250 y $2000 \mathrm{~Hz}$, lo que hace que se diagnostique en etapas tardías ${ }^{41,42}$. En este estudio, cuatro de los cinco pacientes tuvieron estudio audiométrico y tres de ellos $(60 \%)$ presentaban pérdida auditiva de moderada a grave, con una edad promedio al diagnóstico de 16.6 años.

Hasta la fecha, se sabe muy poco sobre el amplio espectro de las alteraciones neurológicas en los pacientes con SW. Previamente se creía que estas alteraciones comenzaban en la tercera década de la vida; sin embargo, hoy se sabe que su inicio es en edades mucho más tempranas ${ }^{43-45}$.

Las complicaciones neurológicas son la principal causa de muerte en los pacientes con SW y su espectro comprende apnea central, ataxia del tronco, neuropatía periférica, pérdida del reflejo nauseoso que predispone a broncoaspiración, mioclonías, pérdida del olfato y del gusto, epilepsia y nistagmo ${ }^{46-48}$. En nuestro estudio se describió alguna alteración neurodegenerativa en cuatro de cinco pacientes, con una edad promedio al diagnóstico de la primera manifestación de $14.25 \pm 7.45$ años, siendo las crisis convulsivas la manifestación más frecuente $(n=2)$. Otras alteraciones descritas en los pacientes de este trabajo son neuropatía periférica, mioclonías, temblor 
postural, anosmia y alteraciones cognitivas; cada una de estas se encontró presente en solo uno de los pacientes.

Las alteraciones psiquiátricas que se observan en los pacientes con SW son muy diversas. El trastorno depresivo es la alteración más frecuentemente encontrada en todas las series, incluyendo la nuestra (80\%); sin embargo, el comportamiento violento y agresivo, la ansiedad, la psicosis, los trastornos bipolares y las conductas impulsivas también son hallazgos frecuentes. El diagnóstico psiquiátrico puede variar con el tiempo en un mismo paciente ${ }^{49,50}$.

Se sugiere que el gen WFS1 tiene un papel en la neuropsicopatología del suicidio y puede ser un biomarcador del estrés postraumático ${ }^{50,51}$; las altas tasas del mismo entre los pacientes con SW hacen que la valoración psiquiátrica sea obligatoria. En nuestra serie, todos los pacientes habían sido atendidos en la consulta de psiquiatría y el $40 \%$ contaban con antecedente de al menos dos intentos suicidas; en todos los casos, la insulina fue utilizada como método de intento de suicidio. Tanto las personas homocigotas como las heterocigotas para mutaciones en WFS1 tienen un aumento del riesgo de suicidio ${ }^{50}$.

Las alteraciones urológicas se presentan aproximadamente en el $58-100 \%$ de los pacientes e inicialmente se atribuían al elevado gasto urinario secundario a la diabetes insípida y la hiperglucemia; sin embargo, la persistencia en la dilatación del tracto urinario a pesar del control de la diabetes insípida y la evidencia disponible de los estudios urodinámicos han demostrado que la disfunción vesical es la causa principal de la hidroureteronefrosis que se observa en estos pacientes $^{3,30,40}$. En nuestra serie, el $100 \%$ de los pacientes presentaron al menos alguna alteración urológica, siendo la vejiga neurogénica la más frecuente $(n=3)$, con una edad promedio al diagnóstico de $13.4 \pm 5.68$ años; dos de los pacientes realizan cateterismo vesical intermitente como parte de su tratamiento.

Las alteraciones gonadales se observan frecuentemente en pacientes del sexo masculino, y de forma muy rara y leve en las mujeres ${ }^{37}$. Aún no se ha logrado dilucidar la razón por la que esta alteración es prácticamente exclusiva del sexo masculino.

En las mujeres, la función ovárica se encuentra preservada y se ha informado únicamente de alteraciones menstruales leves o moderadas, como ocurrió en las dos mujeres incluidas en este estudio; sin embargo, hay muchos casos de mujeres con SW que han presentado embarazos a término, sin complicaciones y con hijos sanos ${ }^{43,52}$. En esta serie, las dos mujeres presentaron irregularidades menstruales, ambas eran nuligestas y no tenían perfil hormonal para su estudio. Como otra alteración endocrinológica, las dos mujeres del estudio contaban con diagnóstico de hipotiroidismo en tratamiento sustitutivo con levotiroxina al momento del estudio.

Los síntomas de hipogonadismo, hipogonadotrófico o hipergonadotrófico, se presentan aproximadamente en el $34 \%$ de los hombres con SW ${ }^{17}$, incluyendo pubertad retrasada, atrofia testicular que causa alteraciones en la forma de los espermatozoides y un número reducido de células espermatogénicas, además de disfunción eréctil, entre otros ${ }^{53}$. En este estudio, el $100 \%$ de los hombres $(n=3)$ contaban con diagnóstico de hipogonadismo hipergonadotrófico por estudios de laboratorio.

El cáncer testicular (seminoma clásico), documentado en uno de nuestros pacientes, no había sido informado previamente en asociación con el SW.

Hasta el $25 \%$ de los pacientes presentan alteraciones en la motilidad gastrointestinal, aunque frecuentemente pasan desapercibidas. El espectro sintomático incluye gastroparesia, incontinencia debido a debilidad en los esfínteres, diarrea y estreñimiento, secundarias a la neurodegeneración de las células en los plexos mientéricos ${ }^{26,33,54}$. En esta serie, dos de los pacientes presentaban estreñimiento crónico, mientras que en uno se realizó el diagnóstico de hepatitis autoinmunitaria con serología y biopsia, asociación no descrita previamente en la literatura.

En series internacionales se ha informado de un gran número de alteraciones cardiacas, entre las que destacan alteraciones valvulares, defectos en el tabique interventricular, tetralogía de Fallot y arritmias, siendo la taquicardia supraventricular paroxística la más frecuente ${ }^{3,27,38}$. En este estudio, ningún paciente tenía una anormalidad cardiaca diagnosticada.

Más del $90 \%$ de los pacientes con SW presentan alguna mutación en uno o en los dos alelos del gen WFS1. En aquellos en quienes no se ha identificado ninguna mutación existe la posibilidad de que esta se encuentre en el promotor (que aún no ha sido codificado), en las secuencias intrónicas o el exón 1 no codificante, ya que no fueron buscados en la mayoría de los estudios $4,41,55$.

Hasta el momento se han descrito más de 200 mutaciones distintas del gen WFS1. La mayoría de las mutaciones son únicas para un individuo o una familia, por lo que su naturaleza privada hace muy difícil el delinear una clara relación genotipo-fenotipo ${ }^{32,56,57}$. Las mutaciones se distribuyen aleatoriamente en toda 
la secuencia codificante del gen, pero hasta el $80 \%$ de las mutaciones reportadas hasta la fecha se encuentran en el exón 825,26,30,31,37,56-59.

En este estudio, el $60 \%$ de los pacientes $(n=3)$ cuentan con diagnóstico molecular y se trata de tres hermanos (los tres hombres del estudio) que presentan dos mutaciones en estado heterocigoto: la primera, heredada del padre, es una transición de guanina por citocina en el codón 177 que cambia una arginina por una prolina en el exón 5 , y la segunda es una deleción de herencia materna de 16 pb (P451fsX515) en el exón 8 que cambia el marco de lectura y genera un codón de terminación prematura en la secuencia normal de la proteína. Estas mutaciones ya habían sido descritas previamente en esta familia por Zenteno, et al. ${ }^{60}$ en 2008.

Estudios de seguimiento sugieren que los pacientes con SW eventualmente desarrollan todo el espectro de la enfermedad si viven lo suficiente. Muchos pacientes con SW pueden permanecer sin diagnóstico hasta la adultez debido al mal diagnóstico de diabetes tipo 1 y a la interpretación errónea de los síntomas clínicos de las alteraciones neurodegenerativas, sobre todo en etapas tempranas, al atribuirlos a complicaciones de la diabetes ${ }^{43}$. Hasta la fecha no existe ninguna intervención que altere la progresión de la enfermedad o prolongue la expectativa de vida en el SW, pero el reconocimiento temprano de este síndrome y sus complicaciones puede incrementar la calidad de vida de quienes la padecen al permitir intervenciones tempranas para los componentes de este síndrome ${ }^{22}$.

Aunque el SW es una enfermedad rara, deberá sospecharse en todos aquellos casos de diabetes mellitus con síntomas de atrofia óptica progresiva, pérdida auditiva, diabetes insípida, síntomas psiquiátricos o alteraciones del tracto urinario ${ }^{43,61}$.

\section{Bibliografía}

1. Wolfram DJ, Wagener HP. Diabetes mellitus and simple optic atrophy among siblings: report of four cases. Mayo Clin Proc. 1938;13:715-8.

2. Rigoli L, Lombardo F, Di Bella C. Wolfram syndrome and WSF1 gene. Clin Genet. 2011;79:103-17.

3. Kumar S. Wolfram syndrome: important implications for pediatricians and pediatric endocrinologists. Pediatric Diabetes. 2010;11:28-37.

4. Khanim F, Kirk J, Latif F, et al. WFS1/Wolframin mutations, Wolfram syndrome, and associated diseases. Human Mutation. 2001;17:357-67.

5. Barret TG. Mitocondrial diabetes, DIDMOAD and other inherited diabetes syndromes. Best Pract Res Clin Endocrinol Metab. 2001;15:325-43.

6. Gómez-Zaera M, Strom TM, Rodríguez B, et al. Presence of a major WFS1 mutation in Spanish Wolfram syndrome pedigries. Mol Genet Metabol. 2001;72:72-81.

7. Fraser FC, Gunn T. Diabetes mellitus, diabetes insipidus, and optic atrophy. An autosomal recessive syndrome? J Med Genet. 1977;14:190-3.

8. Inoue $\mathrm{H}$, Tanizawa $\mathrm{Y}$, Wasson J, et al. A gene encoding a transmembrane protein is mutated in patients with diabetes mellitus and optic atrophy (Wolfram syndrome). Nat Genet. 1998;20:143-8.
9. Hofmann S, Philbrook C, Gerbitz KD, et al. Wolfram syndrome: structural and functional analisys of mutante and wild-type wolframin, the WFS1 gene product. Hum Mol Genet. 2003;12:2003-12.

10. Wang S, Kaufman RJ. The impact of the unfolded protein response on human disease. J Cell Biol. 2012;197:857-67.

11. Fonseca SG, Gromada J, Urano F. Endoplasmic reticulum stress and pancreatic $\beta$-cell death. Trends Endocrinol Metabol. 2011;22:266-74.

12. Zatyka M, Da Silva XG, Bellomo EA, et al. Sarco(endo)plasmic reticulum ATPase is a molecular partner of Wolfram syndrome 1 protein, wich negatively regulates its expression. Hum Mol Genet. 2015;24:814-27.

13. Fonseca SG, Burcin M, Gromada J, et al. Endoplasmic reticulum stress in $\beta$-cells and development of diabetes. Curr Opin Pharmacol. 2009;9:763-70.

14. Araki E, Oyadomari S, Mori M. Endoplasmic reticulum stress and diabetes mellitus. Intern Med. 2003;42:7-14.

15. Araki E, Oyadomari S, Mori M. Impact of endoplasmic reticulum stress pathway on pancreatic $\beta$-cells and diabetes mellitus. Exp Biol Med. 2003;228:1213-7.

16. Mozzillo $E$, Delvecchio $M$, Carella $M$, et al. A novel CISD2 intragenic deletion, optic neuropathy and platelet aggregation defect in Wolfram syndrome type 2. BMC Med Genet. 2014;24:15-88.

17. Rohayem J, Ehlers C, Wiedeman B, et al. Diabetes and neurodegeneration in Wolfram syndrome. Diabetes Care. 2011;34:1503-10.

18. Oslowski CM, Urano F. A switch from life to death in endoplasmic reticulum stressed $\beta$-cells. Diabetes Obes Metab. 2010;12 (Suppl 2):58-65.

19. Ghirardello S, Dusi E, Castiglione B, et al. Congenital central diabetes insipidus and optic atrophy in a Wolfram newborn: is there a role for WFS1 gene in neurodevelopment? Int J Pediatr. 2014;26:40-76.

20. Cnop M, Foufelle F, Velloso LA. Endoplasmic reticulum stress, obesity and diabetes. Trends Mol Med. 2012;18:59-68.

21. Urano F. Wolfram syndrome iPS cells: the first human cell model of endoplasmic reticulum disease. Diabetes. 2014;63:844-6.

22. Marshall BA, Permutt MA, Paciorkowski AR, et al. Phenotypic characteristics of early Wolfram syndrome. Orphanet J Rare Dis. 2013;27:8-64.

23. Urano F. Targeting endoplasmic reticulum to combat juvenile diabetes. Nat Rev Endocrinol. 2014;10:129-30.

24. Barret TG, Bundey SE, Macleod AF. Neurodegeneration and diabetes: UK nationwide study of Wolfram (DIDMOAD) syndrome. Lancet. 1995;346:1458-63

25. Matsunaga K, Tanabe $\mathrm{K}$, Inoue $\mathrm{H}$, et al. Wolfram syndrome in the Japanese population; molecular analysis of WFS1 gene and characterization of clinical features. PLoS One. 2014 Sep 11;9:e106906.

26. Ganie MA, Laway BA, Nisar S, et al. Short report: epidemiology, presentation and course of Wolfram (DIDMOAD) syndrome from North India. Diabet Med. 2011;28:1337-42.

27. Medlej R, Wasson J, Baz P, et al. Diabetes melitus and optic atrophy: a study of Wolfram syndrome in the Lebanese population. J Clin Endocrinol Metab. 2004;89:1656-61.

28. Domènech $E$, Gómez-Zaera $M$, Nunes V. Study of the WFS1 gene and mitocondrial DNA in Spanish Wolfram syndrome families. Clin Genet. 2004;65:463-9.

29. Chaussenot A, Rouzier C, Quere M, et al. Mutation update and uncommon phenotypes in a French cohort of 96 patients with WSF1-related disorders. Clin Genet. 2015;87:430-9.

30. Gasparin MRR, Crispim F, Paula SL, et al. Identification of novel mutations of the WFS1 gene in Brazilian patients with Wolfram syndrome. Eur J Endocrinol. 2009;160:309-16.

31. Aloi C, Saina A, Pasquali L, et al. Wolfram syndrome: new mutations, different phenotype. PLoS One. 2012;7:e29150.

32. López de Heredia M, Clèries R, Nunez V. Genotypic classification of patients with Wolfram syndrome: insights into the natural history of the disease and correlation with phenotype. Genet Med. 2013;15:497-506.

33. Barret TG, Bundey SE. Wolfram (DIDMOAD) syndrome. J Med Genet. 1997;34:838-41.

34. Cano A, Molines L, Valero R, et al., and the French Group of Wolfram Syndrome. Microvascular diabetes complications in Wolfram syndrome (diabetes insipidus, diabetes mellitus, optic atrophy and deafness [DIDMOAD]): an age- and duration-matched comparison with common type 1 diabetes. Diabetes Care. 2007;30:2327-30.

35. Zmyslowska A, Fendler W. Szadkowska A, et al. Glycemic variability in patients with Wolfram syndrome is lower than in type 1 diabetes. Acta Diabetol. 2015;52:1057-62.

36. Barret TG, Bundey SE, Fielder AR, et al. Optic atrophy in Wolfram (DIDMOAD) syndrome. Eye. 1997;11:882-8.

37. Boutzios G, Lavadas S, Marinakis E, et al. Endocrine and metabolic aspects of the Wolfram syndrome. Endocrine. 2011;40:10-3.

38. Smith CJA, Crock PA, King BR, et al. Phenotype-genotype correlations in a series of Wolfram syndrome families. Diabetes Care. 2004;27:2003-9.

39. Gabreels BA, Swaab DF, De Kleijn DP, et al. The vasopressin precursor is not processed in the hypothalamus of Wolfram syndrome patients with diabetes insipidus: evidence of the involvement of PC2 and 7B2. J Clin Endocrinol Metab. 1998;83:4026-33. 
40. Simsek E, Simsek T, Tekgül S, et al. Wolfram (DIDMOAD) syndrome: a multidisciplinary clinical study in nine Turkish patients and review of the literature. Acta Paediatr. 2003;92:55-61.

41. van ven Ouweland JM, Cryns K, Pennings RJ, et al. Molecular characterization of WFS1 in patients with Wolfram syndrome. J Mol Diagn 2003;5:88-95

42. Pennings RJE, Bom SJH, Cryns K, et al. Progression of low-frequency sensorineural hearing loss (DFNA6/14-WFS1). Arch Otolaryngol Head Neck Surg. 2003;129:421-6.

43. Homa K, Stefanski A, Zmylowska A, et al. False diagnosis of type 1 diabetes mellitus and its complications in Wolfram syndrome -is it the reason for the low number of reported cases in this anormality? Endokrynologia Polska. 2014;65:5.

44. Chaussenot A, Bannwarth S, Rouzier $C$, et al. Neurologic features and genotype-phenotype correlation in Wolfram syndrome. Ann Neurol. 2011;69:501-8

45. Waschbisch A, Volbers B, Struffert T, et al. Primary diagnosis of Wolfram syndrome in an adult patient - case report and description of a novel patogenic mutation. J Neurol Sci. 2011;300:191-3.

46. Hershey T, Lugar HM, Shimony JS, et al. Early brain vulnerability in Wolfram syndrome. PLoS One. 2012;7:e40604.

47. Mathis S, Maisonobe T, Neau JP. Neuropathy in Wolfram syndrome. Eur J Med Genet. 2011;54:73-5.

48. Pickett KA, Duncan RP, Paciorkowski AR, et al. Balance impairment in individuals with Wolfram syndrome. Gait Posture. 2012;36:619-24.

49. Swift M, Swift RG. Psychiatric disorders and mutations at the Wolfram syndrome locus. Biol Psychiatry. 2000;47:787-93.

50. Sequeira A, Kim C, Seguin M, et al. Wolfram syndrome and suicide: evidence for a role of WFS1 in suicidal and impulsive behavior. Am J Med Gen B Neuropsychiatr Genet. 2003;119:108-13.

51. Kytövuori L, Seppänen A, Martikainen MH, et al. WFS1 variants in Finnish patients with diabetes mellitus, sensorineural hearing impairment or optic atrophy, and in suicide victims. J Hum Genet. 2013:58:495-500.
52. Sobhami M, Tabatabaifar MA, Rajab A, et al. Significant expressivity of Wolfram syndrome: phenotypic assesement of two known and one novel mutation in the WFS1 gene in three Iranian families. Mol Biol Rep. 2014;41:7499-505.

53. Haghighi A, Haghighi A, Setoodeh A, et al. Identification of homozygous WFS1 mutations (p.Asp211Asn, p.Gln486*) causing severe Wolfram syndrome and first report of male fertility. Eur J Hum Genet. 2013;21:347-51.

54. Lui Z, Sakakibara R, Uchiyam T, et al. Bowel dysfunction in Wolfram syndrome. Diabetes Care. 2006;29:472-3.

55. Hardy C, Khanim F, Torres R, et al. Clinical and molecular genetic analysis of 19 Wolfram syndrome kindreds demonstrating a wide spectrum of mutations in WFS1. Am J Hum Genet. 1999;65:1279-90.

56. D'Annunzio G, Minuto N, D'Amato E, et al. Wolfram syndrome (diabetes insipidus, diabetes, optic atrophy, and deafness). Clinical and genetic study. Diabetes Care. 2008;31:1743-45.

57. Cryns K, Sivakumaran TA, Van den Ouweland JM, et al. Mutational spectrum of the WFS1 gene in Wolfram syndrome, nonsyndromic hearing impairment, diabetes mellitus and psychiatric disease. Human Mutation. 2003;22:275-87.

58. Yu G, Yu M, Wang J, et al. WS1 gene mutation analysis of Wolfram syndrome in a Chinese patient and a systematic review of literature. Endocrine. 2010;38:147-52.

59. Sobhani M, Tabatabaiefar MA, Rajab A, et al. Molecular characterization of WFS1 in an Iranian family with Wolfram syndrome reveals a novel frameshift mutation associated with early symptoms. Gene. 2013;528:309-13.

60. Zenteno JC, Ruiz G, Pérez-Cano HJ, et al. Familial Wolfram syndrome due to compound heterozygosity for two novel WFS1 mutations. Mol Vis. 2008;14:1353-7.

61. Blanco-Aguirre ME, la Parra DR, Tapia-Garcia $\mathrm{H}$, et al. Identification of unsuspected Wolfram syndrome cases through clinical assessment and WFS1 gene screening in type 1 diabetes mellitus patients. Gene. 2015;15:63-7. 